Letter

\section{Off-label Use of Secukinumab: A Potential Therapeutic Option for SAPHO Syndrome}

\section{To the Editor:}

We read the recent article by Wang et al with great interest. ${ }^{1}$ The authors described a cohort of 4 patients with SAPHO (synovitis, acne, pustulosis, hyperostosis, and osteitis) syndrome who showed substantial improvement in skin lesions, clinical conditions, and whole-body magnetic resonance imaging before and after treatment with secukinumab without concomitant conventional synthetic disease-modifying antirheumatic drugs (csDMARDs), nonsteroidal antiinflammatory drugs (NSAIDs), or other biologics, and suggested a potential benefit of secukinumab in the treatment of SAPHO syndrome. However, there are some details that need further clarification.

First, SAPHO syndrome is a rare chronic inflammatory disease involving bone, joint, and skin. ${ }^{2}$ Although variable degrees of the efficacy of pharmacological therapies including antibiotics, bisphosphonates, NSAIDs, glucocorticoids, DMARDs, interleukin (IL)-1 receptor antagonist, and anti-tumor necrosis factor (TNF) have been described previously, ${ }^{2}$ there is currently no consensus on the treatment of SAPHO syndrome and different treatment options may lead to different outcomes. Second, recent studies have revealed the potential role of cytokine dysregulation in SAPHO syndrome, such as IL-1 $\beta$, TNF- $\alpha$, IL-8, IL-17, IL-18, and activated neutrophils. ${ }^{3}$ Blocking these cytokines may be an effective strategy for the treatment of SAPHO syndrome. ${ }^{3}$ IL-17 is a cytokine produced by Th17 cells, and a rising number of studies have identified a pathogenic role for IL-17 in SAPHO syndrome. For synovitis, IL-17 is effective in peripheral arthritis in the context of spondyloarthritis. For acne, the expression of IL-17 in acne lesions is increased and infiltrated by a large number of IL-17-expressing $\mathrm{T}$ cells. In addition, Propionibacterium acnes can trigger IL-17 secretion from CD4+ $\mathrm{T}$ cells in vitro. For pustulosis, IL-17 expression is increased in the skin of palmoplantar pustulosis. For hyperostosis, IL-17 induces osteoblastogenesis in vitro, whereas in an animal model of $\mathrm{SpA}$, it inhibits new bone formation. For osteitis, inhibition of IL-17 is effective in suppressing spinal inflammation in ankylosing spondylitis (AS). ${ }^{4}$ Secukinumab is a fully human $\operatorname{IgG} 1 / \kappa$ monoclonal antibody that specifically binds IL-17A and inhibits its interaction with the IL-17 receptor, thereby reducing IL-17A-mediated inflammatory disease, and has been approved for the treatment of psoriatic arthritis, moderate-to-severe psoriasis $(\mathrm{PsO}), \mathrm{AS}$, hypertrophic palmoplantar $\mathrm{PsO}$, and generalized pustular PsO.5 Given its potent inhibition of IL-17 expression, secukinumab is being increasingly used off-label for the rheumatic diseases, particularly for those that are refractory to current standard treatment algorithms, including systemic lupus erythematosus, rheumatoid arthritis, TNF receptor-associated periodic syndrome, and familial Mediterranean fever. ${ }^{6,7}$ Finally, the study by Wang et $\mathrm{al}^{1}$ does provide an important therapeutic option for patients with SAPHO syndrome using secukinumab, but further observations are needed due to the limitations in sample size and design. In addition, given the complexity and heterogeneity of SAPHO syndrome, identifying possible benefits from secukinumab treatment merits consideration.

In summary, although some of the details in this article need to be further elucidated, this study reveals successful cases of secukinumab in the treatment of SAPHO syndrome and supports the growing interest in the use of secukinumab for SAPHO syndrome.

\section{Gang Wang ${ }^{1}$, MD}

Ning Zhuo $^{2}$, MD

Jingyang $\mathrm{Li}^{1}(\mathbb{D}, \mathrm{MD}$

${ }^{1}$ Department of Rheumatology and Immunology, Zhuzhou Hospital Affiliated to Xiangya Medical College, Central South University, Zhuzhou;

${ }^{2}$ Department of Nephrology, Xiangya Second Hospital, Central South University, Changsha, Hunan, China.

The authors declare no conflicts of interest relevant to this article.

Address correspondence to Dr. J. Li, Department of Rheumatology and Immunology, Zhuzhou Hospital Affiliated to Xiangya Medical College, Central South University, Zhuzhou, Hunan, China.

Email:wgzncsu@csu.edu.cn.

\section{ACKNOWLEDGMENT}

We would like to thank the members and staff of the Department of Rheumatology and Immunology of the Zhuzhou Central Hospital who contributed to this manuscript.

\section{REFERENCES}

1. Wang L, Sun B, Li C. Clinical and radiological remission of osteoarticular and cutaneous lesions in SAPHO patients treated with secukinumab: a case series. J Rheumatol 2021;48:953-5.

2. Firinu D, Garcia-Larsen V, Manconi PE, et al. SAPHO Syndrome: current developments and approaches to clinical treatment. Curr Rheumatol Rep 2016;18:35.

3. Liu S, Tang M, Cao Y, et al. Synovitis, acne, pustulosis, hyperostosis, and osteitis syndrome: review and update. Ther Adv Musculoskelet Dis 2020;12:1759720x20912865.

4. Daoussis D, Konstantopoulou G, Kraniotis P, et al. Biologics in SAPHO syndrome: a systematic review. Semin Arthritis Rheum 2019;48:618-25.

5. Braun J, Kiltz U, Bühring B, et al. Secukinumab in axial spondyloarthritis: a narrative review of clinical evidence. Ther Adv Musculoskelet Dis 2021;13:1759720x211041854.

6. Kerschbaumer A, Sepriano A, Smolen JS, et al. Efficacy of pharmacological treatment in rheumatoid arthritis: a systematic literature research informing the 2019 update of the EULAR recommendations for management of rheumatoid arthritis. Ann Rheum Dis 2020;79:744-59.

7. Cannavò SP, Papaianni V, Bartolotta A, et al. Secukinumab for psoriasis in a patient with familial Mediterranean fever. Dermatol Therapy 2019;32:e13122. 